FORMATION Formation emploi

Revue française de sciences sociales

106 | avril-juin 2009

Pêle-mêle

\title{
De l'influence du parcours de formation sur l'insertion : le cas des diplômés scientifiques
}

Vom Einfluss des Ausbildungsgangs auf die berufliche Eingliederung am Beispiel von wissenschaftlichen Diplomen

The influence of training pathways on securing employment: the case of science graduates

De la influencia del itinerario de formación sobre la inserción : el caso de los graduados científicos

\section{Catherine Béduwé, Bernard Fourcade et Jean-François Giret}

\section{OpenEdition}

Journals

Édition électronique

URL : http://journals.openedition.org/formationemploi/1886

DOI : 10.4000/formationemploi.1886

ISSN : 2107-0946

Éditeur

La Documentation française

Édition imprimée

Date de publication : 1 juin 2009

Pagination : 5-22

ISSN : 0759-6340

Référence électronique

Catherine Béduwé, Bernard Fourcade et Jean-François Giret, « De l'influence du parcours de formation sur l'insertion : le cas des diplômés scientifiques », Formation emploi [En ligne], 106 | avril-juin 2009, mis en ligne le 01 juin 2011, consulté le 30 octobre 2020. URL : http://journals.openedition.org/ formationemploi/1886 ; DOI : https://doi.org/10.4000/formationemploi.1886

(ㄷ) Tous droits réservés 


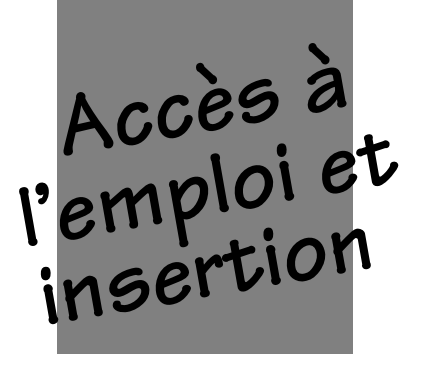

\section{De l'influence du parcours de formation sur l'insertion : le cas des diplômés scientifiques}

Catherine Béduwé, Bernard Fourcade et Jean-François Giret ${ }^{\star}$

\section{Focaliser l'attention sur les performances d'insertion du seul diplôme terminal, sans prendre en compte les éléments du parcours préalable, s'avère une simplification. Le niveau du diplôme terminal ainsi que sa spécialité sont certes majeurs pour l'insertion mais, à diplôme égal, le parcours de formation joue un rôle important.}

\begin{abstract}
À l'heure où la loi LRU (Libertés et responsabilités des universités), du 11 août 2007, invite les universités à mieux lutter contre l'échec en cours d'études en diversifiant davantage les parcours de formation des étudiants, mais aussi à renforcer leur information sur les performances d'insertion des diplômes qu'elles délivrent, il semble opportun de s'interroger sur les effets propres des parcours sur les performances d'insertion, au-delà des effets bien connus du diplôme lui-même et surtout de son niveau.
\end{abstract}

Au moment d'entamer leurs études supérieures, les bacheliers ont, depuis longtemps, le choix entre plusieurs types de filières qui sont le DEUG à l'université, l'IUT, les STS, une $\mathrm{CPGE}^{1}$, choix qui peut dépendre de leurs capacités. Les possibilités de passages entre filières existent déjà mais la nouvelle

${ }^{1}$ Respectivement DEUG : diplôme d'études universitaires générales ; IUT : Institut universitaire de technologie ; STS : Section de technicien supérieur ; CPGE : Classe préparatoire aux Grandes Écoles.
Catherine Béduwé est ingénieur de recherches au LIRHE (Laboratoire interdisciplinaire de recherche sur les ressources humaines et l'emploi), à l'université de Toulouse. Ses travaux portent sur l'insertion professionnelle des jeunes et la relation formation-emploi. Elle a co-coordonné une recherche européenne, (2004), "The effects of educational expansion on the functioning of the labour market: Report of a comparative study ", with J. Planas, Review Compare (Taylor \& Francis Group), vol. 34, n 1, 2004, pp. 54-71. Publication récente en commun avec les deux autres auteurs: "Études scientifiques et marché du travail ", (2007) in L'orientation scolaire et professionnelle, vol. 36, $n^{\circ} 4$, pp. 503-532.

Bernard Fourcade est ingénieur de recherches au LIRHE. Ses travaux récents portent sur les politiques de formation professionnelle et de certification, et sur le développement des observatoires de l'emploi et de la formation dans les pays partenaires de l'Union européenne. 
Jean-François Giret est maître de conférences en sciences économiques à l'université de Bourgogne et chercheur à l'IREDU (Institut de recherche sur l'éducation). Ses travaux, à la croisée de l'économie de l'éducation et du travail, portent sur l'analyse de la relation formation-emploi, I'insertion professionnelle des jeunes et les carrières des chercheurs.

loi incite les universités à développer l'orientation des étudiants, de manière à ce que chaque étudiant suive le parcours de formation qui convient le mieux à ses aptitudes afin qu'il obtienne un diplôme et donc un emploi. Chaque université sera ainsi de plus en plus souvent amenée à délivrer un diplôme de licence ou de master à des étudiants issus d'horizons divers et qui n'ont pas suivi les mêmes enseignements.

Cette évolution contribue à déconnecter le processus de formation de sa certification. Les parcours sont jugés équivalents par le système universitaire, puisque le diplôme certifie l'acquisition des compétences, mais est-ce bien certain? Le marché du travail est-il indifférent à la nature du parcours de formation d'un jeune débutant? Ces parcours n'émettent-ils pas des signaux classants ou n'apportent-ils pas des compétences, des expériences supplémentaires valorisables sur le marché ? Si tel était le cas, les détenteurs d'un même diplôme universitaire obtenu au terme de parcours différents dans l'enseignement supérieur n'auront pas les mêmes performances d'insertion professionnelle. Comment alors juger de la performance d'insertion d'un diplôme donné et quelle information fournir aux étudiants?

L'analyse est complexe pour au moins deux raisons. La première est qu'il existe à l'évidence des liens entre le parcours de formation, le niveau de diplôme atteint et la spécialité de formation choisie, et qu'aucun de ces éléments n'est indépendant des capacités scolaires du jeune, elles-mêmes en partie déterminées, on le sait, par l'origine sociale. Autrement dit, non seulement l'école ne gomme pas toutes les inégalités sociales mais elle peut même les amplifier au travers des choix de cursus de formation. La seconde raison vient de ce que la mesure des performances d'insertion professionnelle exige l'utilisation de plusieurs indicateurs. Tester l'impact des parcours de formation sur l'insertion, au-delà des effets croisés de diplôme, de spécialité et d'origine sociale nécessite donc quelque prudence mais l'enjeu nous a semblé suffisamment important pour qu'on s'y risque. Il s'agit de premiers résultats qui devront être étendus et confirmés.

Ces questions ont été jusqu'ici assez peu abordées. Quelques travaux, tant en France qu'à l'étranger, ont cependant montré qu'ignorer le parcours de formation (student's educational path) peut conduire à surestimer les rémunérations attachées à un diplôme donné. Des travaux américains (Hilmer, 2006), français (Adangnikou, 2007) et hollandais (Van der Velden, Wolbers, 2007) sur l'enseignement supérieur, ou encore français sur l'enseignement secondaire (Dupray, Recotillet, 2005 ; Dauty, Lemistre, 2008) ont, par exemple, validé ces hypothèses.

Afin de faciliter l'interprétation de nos résultats statistiques, nous avons choisi de restreindre l'analyse à la population spécifique des diplômés en sciences sortis de l'université. Méthodologiquement, ce choix se justifie du fait de l'existence de plusieurs filières au sein des études supérieures scientifiques (la filière des CPGE, celle des universités ou celle des IUT - voire STS), et donc de la possibilité, après réorientation ou poursuite d'études, d'obtenir un même diplôme universitaire (dès bac +3$)$ après des parcours bien différenciés. Par ailleurs, les travaux menés de longue date sur ces diplômés universitaires scientifiques ont montré l'importance des effets croisés du niveau et de la spécialité du diplôme terminal sur l'insertion et les débouchés professionnels (Pottier, 1985; Charlot et Pottier 1989; Béduwé, Giret, Fourcade, Moullet, 2007).

L'étude a été conduite sur les diplômés ayant terminé leur formation initiale en 1998, soit auprès de générations d'étudiants formés avant la mise en place du LMD (licence-master-doctorat ${ }^{2}$ ) dans les universités. Mais le mouvement de diversification des parcours était déjà largement entamé dans les années 90, avec la multiplication des filières, des bifurcations et des passerelles. Après avoir discuté la notion de parcours de formation dans l'enseignement supérieur, nous montrerons son impact sur l'insertion des diplômés en sciences.

\footnotetext{
${ }^{2}$ LMD : nouvelles normes universitaires européennes qui distinguent trois blocs de formation au-delà du bac (3-5-8 ans).
} 


\section{LE PARCOURS DE FORMATION : UN CRITĖRE DE SÉLECTION QUE LE JEUNE INCLUT DANS SON CV ?}

Ayant défini ce que l'on entend par parcours de formation dans le cas des étudiants en sciences, on montrera d'une part l'intérêt d'en tenir compte dans l'analyse de l'insertion professionnelle puis, à l'aide des données de l'enquête Génération 98 du Céreq, d'autre part que le même diplôme scientifique peut être effectivement acquis à l'issue de parcours diversifiés.

\section{Pourquoi s'intéresser} aux parcours de formation?

Le parcours de formation désigne le cheminement éducatif d'un individu depuis la fin de l'école obligatoire jusqu'au moment où il quitte le système de formation initiale, détenteur ou non d'un (ou de plusieurs) diplôme(s). En France, ces parcours sont largement individuels, tout en étant fortement contraints par la structure de l'offre de formation. Dans l'enseignement supérieur, les étudiants peuvent choisir chaque étape de leur parcours mais sous la contrainte que l'accès aux diverses filières ou formations est plus ou moins sélectif. Le mode et le niveau de sélectivité de ces filières constituent une dimension majeure de la caractérisation des parcours.

En sciences tout particulièrement, plusieurs types de filières coexistent à l'entrée dans l'enseignement supérieur: la filière très sélective des CPGE, les écoles (d'ingénieur, de santé...) qui recrutent sur dossier au niveau du bac, les deux filières technologiques courtes (IUT ou STS) à accès plus ou moins sélectif, sur dossier ou sur examen d'entrée, la première année de médecine, ouverte en principe à tous les bacheliers mais pour laquelle existe une forte auto-sélection, et la filière universitaire, à accès libre, mais où les premières années se caractérisent par un fort taux d'échec aux examens. Il existe, entre ces filières, de nombreuses possibilités de passages qui s'opèrent le plus souvent après l'obtention d'un diplôme (BTS, DUT, diplôme d'ingénieur...), ce qui conduit certains jeunes à accumuler des doubles certifications, universitaires et non universitaires. Ceci constitue une autre dimension importante de la caractérisation des parcours.
Rarement composé de segments "non scientifiques", le parcours peut être constitué d'enseignements scientifiques plus ou moins spécialisés, dont l'accès peut s'avérer également plus ou moins sélectif et qui peuvent, en plus, s'accompagner de changements de type de filière. Ainsi le titulaire d'un diplôme universitaire en physique fondamentale a pu commencer ses études dans l'enseignement technologique court (STS ou IUT) ou encore dans une école d'ingénieurs recrutant au niveau du bac.

$\mathrm{Au}$ total, plus le parcours est long, plus il a des chances d'être composé de changements de filière et/ ou de dominante scientifique, d'être marqué par des diplômes de natures différentes et, corollaire, plus le niveau du diplôme terminal est élevé, plus les parcours peuvent avoir été complexes (Giret, 2003).

Quels cadres théoriques mobiliser pour expliquer le rôle du parcours de formation dans le processus d'insertion professionnelle ? En économie, on peut recourir à deux ensembles principaux : la théorie du capital humain et la théorie du signal et du filtre.

Du côté du «capital humain », on considère que le processus de formation participe à la construction des compétences. Des parcours différents impliquent l'acquisition de compétences plus ou moins distinctes dues aux disciplines, concepts, notions, savoirs, méthodologies inculqués ainsi qu'aux pédagogies et méthodes de travail utilisées. Certaines de ces compétences, considérées comme plus productives par les employeurs, seront mieux valorisées sur le marché du travail, en termes d'accès à l'emploi ou de rémunération (Meng et Heijke, 2005 ; Garcia et Van der Velden, 2008). Les aspects du parcours qui portent sur la nature des savoirs, au travers notamment des choix à dominante scientifique, relèvent de cette hypothèse.

Sous cet angle, les parcours empruntant des filières différentes génèrent des compétences différentes, moins sur le plan conceptuel - encore qu'il y ait des différences marquées entre les CPGE, les IUT et les universités sur le niveau d'approche théorique et l'outillage conceptuel mobilisés - que méthodologique du fait notamment des formes d'encadrement et de la structuration des enseignements qui y dominent.

Du côté du «signal», les étapes du parcours de formation telles que le passage par une filière 
sélective (IUT, STS, école d'ingénieurs recrutant au niveau bac), l'obtention de diplômes connotés positivement (diplôme d'ingénieurs, BTS, DUT) ou la pluri-certification (diplôme universitaire combiné avec d'autres diplômes) font partie de l'information émise et échangée lors du processus de recrutement, et constituent une part du « signalement», en apportant aux recruteurs des informations discriminantes. Il en est de même pour le passage par une CPGE qui indique que le jeune a été fortement sélectionné à l'entrée de l'enseignement supérieur' ${ }^{3}$.

Le parcours (filières fréquentées et diplômes successifs obtenus) est généralement « signalé » à l'employeur dans le CV. Les entreprises françaises, à la différence des entreprises anglaises ou espagnoles, établissent un filtrage serré des candidats sur une large batterie de critères de sélection (Marchal, Rieucau, 2005). Les candidats à une offre d'emploi développent une stratégie rédactionnelle pour leur $\mathrm{CV}$, en choisissant d'inscrire les éléments de leur parcours de formation ou professionnel jugés les plus appréciés des employeurs. Dans le cas des débutants, l'employeur ne saurait être indifférent au parcours dans l'enseignement supérieur. Il est intéressant de noter que lors du recrutement par un cabinet spécialisé, les premiers éléments de sélection se basent sur le diplôme, l'expérience puis sur des informations comme le sexe, l'âge ou la localisation qui sont parfois utilisées de manière discriminatoire. Comme le remarquent Eymard-Duvernay et Marchal (2000), l'enjeu pour les candidats est alors d'amener le recruteur à prendre également en compte d'autres éléments du CV, comme le parcours de formation.

De son côté, la théorie économique du filtre laisse penser que les entreprises ont, à tord ou à raison, une préférence pour tout signal renvoyant à des formations sélectives qui sont réputées révéler des qualités ou des capacités qu'elles valorisent (efficacité, rapidité, réactivité, etc.), et/ou qui leur garantissent une plus grande «homogénéité » des diplômés. La meilleure insertion des diplômés en sciences appliquées correspondrait ainsi au fait que ces disciplines

\footnotetext{
${ }^{3}$ Même si, comme le montre Adangnikou (2007), ces élèves, une fois entrés dans des écoles d'ingénieurs à recrutement mixte, n'y réussissent pas mieux que les autres.
}

sont le plus souvent enseignées dans des formations sélectives, y compris à l'université.

Ces différentes hypothèses n'excluent pas d'autres explications en sociologie, comme celle des réseaux socioprofessionnels. Les liens créés par l'institution scolaire ou universitaire ainsi que les relations sociales ou professionnelles éloignées (souvent qualifiées de liens faibles en sociologie), mais néanmoins liées au capital social des jeunes, occupent en France une place déterminante pour l'accès aux professions les plus qualifiées (Forsé, 2001). Le développement des filières professionnelles dans l'enseignement supérieur, sélectives et technologiques, a pu accentuer ce type d'intermédiation. C'est notamment le cas des jeunes passés par les IUT qui ont pu nouer des relations professionnelles au cours de stages ou grâce à la présence d'intervenants professionnels (Giret et Moullet, 2008).

Mais peut-on vraiment démêler ce qui relève de ces différents cadres d'interprétation? Qu'il s'agisse des éléments de contenu des formations (les compétences et connaissances acquises), des éléments qui constituent le signal, ou des différentes informations qui ont affecté le processus de recrutement, ils s'avèrent particulièrement difficiles à repérer dans des enquêtes quantitatives. Les enquêtes plus qualitatives ou qui portent sur des échantillons plus spécifiques fournissent des informations complémentaires tout en relativisant ces cadres théoriques. S'il semble acquis que la logique du titre est souvent dominante en France pour les diplômés des grandes écoles d'ingénieurs, il en va autrement pour les diplômés des écoles d'ingénieurs moins prestigieuses et ceux de l'université (Bouffartigue et Gadea, 1997). Dans le cas des SSII (sociétés de service en ingénierie informatique), par exemple, Lanciano (1996) a montré que les ingénieurs étaient souvent recrutés parmi les diplômés de filières scientifiques générales et que l'acquisition des compétences spécifiques à l'emploi se faisait dans l'entreprise. Dans la fonction de R\&D (recherche et développement), la logique semble différente: Duhautois et Maublanc (2005) montrent que les employeurs qui recrutent des chercheurs en entreprise ont des préjugés sur les parcours académiques et privilégient des embauches de diplômés d'écoles d'ingénieurs du fait de leurs compétences plus transversales, mais également en 
raison de logiques de réseaux. De plus, aux spécificités de chaque profession et de chaque fonction s'ajoutent des logiques d'entreprises. Comme le souligne Pottier (1996), à partir de données sur les ingénieurs diplômés du CNAM (Conservatoire national des arts et métiers), le type d'entreprise et ses modes d'organisation du travail vont avoir un effet déterminant sur le recrutement, la promotion et l'affectation à différents emplois.

\section{L'enquête Génération 98 : six parcours de formation chez les diplômés scientifiques}

Repérer les parcours de formation et mesurer leur effet sur l'insertion professionnelle nécessite de disposer de données longitudinales rétrospectives. En effet, il ne s'agit pas de calculer des taux de poursuite d'études à l'issue de chaque diplôme, comme peut le faire le ministère de l'Éducation nationale, mais bien de retracer, a posteriori, le parcours emprunté par un étudiant, une fois ses études achevées et son diplôme terminal atteint. La définition et le repérage de ces parcours vont alors dépendre des éléments disponibles dans l'enquête.

L'exercice a été tenté à l'aide des données de l'enquête Génération 98 du Céreq, qui comporte un échantillon important et représentatif au niveau national des titulaires d'un diplôme scientifique de l'université, supérieur ou égal à la licence, sortis en 1998 et interrogés 3 ans plus tard sur leur insertion professionnelle. Les informations sur le parcours dont on dispose portent sur le type de filière choisi après le bac et sur l'obtention d'un diplôme hors université : BTS, DUT ou diplôme d'ingénieur. En les recoupant, on a pu reconstituer et mettre en évidence six parcours de formation différents chez les titulaires d'un diplôme universitaire de sciences ( $c f$. encadré 1).

\section{Encadré 1}

\section{Les parcours de formation des diplômés en sciences de l'université sortis en 1998}

\section{Les données}

Les données sont issues de l'enquête Génération 98 du Céreq, lancée auprès des sortants du système de formation initiale en 1998, interrogés en 2001 sur leurs trois premières années de vie professionnelle. L'analyse porte sur les titulaires d'un diplôme universitaire en sciences de niveau supérieur ou égal à la licence, hors médecine : licence, maîtrise, DESS et DEA, ces derniers pouvant avoir éventuellement poursuivi quelques années en thèse mais sans obtenir le doctorat. Sont donc exclus tous les diplômes d'ingénieur, aussi bien diplômes d'université que d'École, petite ou grande, ainsi que les sortants d'IUFM (Institut universitaire de formation des maîtres).

On dispose de 1765 questionnaires représentant 15968 individus.

\section{Le repérage des parcours}

Les parcours de formation dans l'enseignement supérieur de ces sortants de l'université ont été reconstitués à partir de deux informations disponibles dans le questionnaire : le type de filière suivi juste après le bac et les éventuels diplômes obtenus en sus de leur diplôme terminal, à savoir BTS, DUT ou diplôme d'école d'ingénieur. En croisant ces deux éléments, on parvient à reconstituer - de manière empirique six types de parcours de formation empruntés par nos diplômés scientifiques.

Parcours 1 : Universitaires purs. Ces étudiants sont entrés dans l'enseignement supérieur par une inscription en DEUG et ont (vraisemblablement) fait toutes leurs études à l'université. Ils n'ont que les diplômes universitaires leur ayant permis d'accéder au niveau supérieur. L'accès à l'université étant libre, ces étudiants n'ont pas été sélectionnés à l'entrée dans l'enseignement supérieur. Ils l'ont été, en revanche, et parfois fortement, tout au long de leurs études. 


\section{Encadré 1 (suite)}

Parcours 2 : IUT et DUT puis université. Après un 1 er cycle à l'IUT, où ils ont obtenu un DUT, ces étudiants ont poursuivi leurs études à l'université. II s'agit, pour certains, d'un " contournement » du ler cycle universitaire via le supérieur court. La sélection à l'entrée des IUT est variable, pas toujours très sévère, du moins pour les titulaires d'un bac $S$.

Parcours 3 : STS et BTS puis université. Après un 1 er cycle en STS et l'obtention de leur BTS, ces étudiants ont poursuivi leurs études à l'université. La sélection à l'entrée y est moins sévère qu'en IUT, accessible notamment pour les titulaires d'un bac technologique.

Parcours 4 : 7re année sélective (hors IUT et STS) et autre diplôme, puis université : Ces étudiants sont entrés dans l'enseignement supérieur par une filière " sélective » autre que I'IUT ou la STS ICPGE, PCEM - respectivement classe préparatoire aux Grandes Écoles et premier cycle études médicales école d'ingénieur niveau bac) et déclarent, par ailleurs, avoir obtenu un diplôme, DUT ou BTS, mais plus souvent un diplôme d'ingénieur. Ce parcours, le moins fréquent, est aussi le plus complexe et les éléments dont on dispose ne sont pas suffisants pour bien le décrire.

Parcours 5 : CPGE puis université. Après avoir passé une ou deux années en CPGE, ces étudiants ont rejoint l'université, après abandon de la filière CPGE, ou échec aux concours.

Parcours 6 : 1 re année à accès sélectif hors CGPE. Ces étudiants sont entrés dans l'enseignement supérieur par une filière sélective (IUT, STS, PCEM, école d'ingénieur niveau bac) mais ont ensuite rejoint l'université, sans avoir obtenu de diplôme. Ce parcours a été distingué du précédent au motif que la sélection en CPGE est beaucoup plus forte. Pour les PCEM, il s'agit même d'auto-sélection plus que de sélection.

On dispose par ailleurs de quelques informations sur la manière dont a pu se dérouler le parcours scolaire de nos diplômés avant le bac : année d'entrée en be, type de seconde (générale ou non), année du bac et type de bac (série).

Sigles : DEUG : diplôme d'études universitaires générales ; DEA : diplôme d'études approfondies ; DESS : diplôme d'études supérieures spécialisées ; IUT : Institut universitaire de technologie ; STS : section de technicien supérieur ; BTS : brevet de technicien supérieur ; DUT : diplôme universitaire de technologie.

On constate tout d'abord que plus de la moitié des titulaires d'un diplôme universitaire de $2^{\mathrm{e}}$ ou $3^{\mathrm{e}}$ cycle en sciences (hors doctorat) ont effectué une part plus ou moins longue de leur parcours en dehors de l'université (tableau 1). Si on distingue ces diplômés par la spécialité de leur diplôme final, en différenciant sciences fondamentales (SF) et sciences appliquées $(\mathrm{SA})^{4}$, on observe que $33 \%$ des diplômés en sciences fondamentales et $61 \%$ des diplômés en sciences appliquées ont effectué une partie de leur parcours en dehors de l'université Ainsi, et contrai-

\footnotetext{
${ }^{4}$ Par convention, les sciences fondamentales regroupent les disciplines académiques de maths, physique, chimie, sciences de l'univers et sciences de la vie et les sciences appliquées relèvent d'une spécialité technique ou industrielle, y.c Informatique, à l'exclusion des formations de la santé.
}

rement à ce que l'on aurait pu penser, même les sortants de l'université dotés d'un diplôme en sciences fondamentales ont pu connaître des parcours « complexes », avec des changements de filière et/ou de domaine de spécialité scientifique.

On constate ensuite que la diversité des parcours existe, quel que soit le diplôme terminal obtenu (licence, maîtrise, DEA et DESS), mais on vérifie que plus les études ont été longues et le diplôme terminal atteint de haut niveau, plus les parcours risquent d'avoir été complexes.

Il existe à l'évidence, lorsque l'on analyse en détail le tableau 1, une corrélation entre le type de parcours de formation et la spécialité finale du diplôme obtenu, ceci étant vrai quel que soit le niveau finalement atteint. Les 
Tableau 1

Type de parcours en fonction du diplôme de sortie

\begin{tabular}{|l|c|c|c|c|c|c|}
\hline \multicolumn{7}{|c|}{ Sciences fondamentales (SF) } \\
\hline \multicolumn{1}{|c|}{ Parcours dans l'enseignement supérieur } & Plus haut diplôme obtenu en sciences fondamentales & $\begin{array}{c}\text { Total } \\
\text { des SF } \\
(\%)\end{array}$ \\
\hline Universitaires purs & 61 & 67 & 62 & 72 & 66 \\
\hline IUT et DUT, puis fac & 8 & 16 & 13 & 6 & 11 \\
\hline STS et BTS, puis fac & 2 & 2 & 8 & 5 & 5 \\
\hline $\begin{array}{l}\text { 1re année sélective (hors IUT et STS) ET autre } \\
\text { diplôme, puis fac }\end{array}$ & 9 & 4 & 1 & 1 & 3 \\
\hline CPGE puis fac & 13 & 6 & 6 & 6 & 7 \\
\hline $\begin{array}{l}\text { 1re sélective (PCEM, IUT, école ingénieur, santé...) } \\
\text { puis université }\end{array}$ & 7 & 5 & 10 & 9 & 8 \\
\hline Total diplômés en SF & 100 & 100 & 100 & 100 & 100 \\
\hline
\end{tabular}

\begin{tabular}{|l|c|c|c|c|c|}
\hline \multicolumn{7}{|c|}{ Sciences appliquées (SA) } \\
\hline \multicolumn{1}{|c|}{ Parcours dans l'enseignement supérieur } & Plus haut diplôme obtenu en sciences appliquées & $\begin{array}{c}\text { Total } \\
\text { des SA } \\
\text { (\%) }\end{array}$ \\
\hline Universitaires purs & 41 & 51 & 30 & 27 & 39 \\
\hline IUT et DUT, puis fac & 16 & 29 & 44 & 31 & 32 \\
\hline STS et BTS, puis fac & 8 & 7 & 13 & 26 & 14 \\
\hline $\begin{array}{l}\text { 1re année sélective (hors IUT et STS) ET autre } \\
\text { diplôme, puis fac }\end{array}$ & 25 & 4 & 3 & 7 & 7 \\
\hline CPGE puis fac & 10 & 5 & 7 & 3 & 6 \\
\hline $\begin{array}{l}\text { 1re sélective (PCEM, IUT, école ingénieur, santé...) } \\
\text { puis fac }\end{array}$ & - & 4 & 3 & 6 & 3 \\
\hline Total diplômés en SA & 100 & 100 & 100 & 100 & 100 \\
\hline
\end{tabular}

Source : Enquête Génération 98 à 3 ans, Céreq.

Par convention, les sciences fondamentales (SF) regroupent les disciplines académiques de maths, physique, chimie, sciences de l'univers et sciences de la vie et les sciences appliquées (SA) celles qui relèvent d'une spécialité technique ou industrielle, y.c informatique, à l'exclusion des formations de la santé.

Sigles : DEA : diplôme d'études approfondies ; DESS : diplôme d'études supérieures spécialisées. DUT : diplôme universitaire de technologie (obtenu à l'issue d'une formation en IUT); BTS ; brevet de technicien supérieur (obtenu à l'issue d'une formation en STS) ; PCEM : Première année commune aux formations de Médecin, Chirurgien Dentiste et Sage Femme ; CPGE : classe préparatoire aux Grandes Écoles.

Note de lecture : 61 \% des diplômés d'un DEA en sciences fondamentales ont eu un parcours purement universitaire.

diplômés en SA ont plus souvent «contourné» le $1^{\text {er }}$ cycle universitaire et poursuivi leurs études en fac après un BTS, un DUT ou même un diplôme d'ingénieur pour les DEA. Cette pratique se fait plus rare pour les sortants diplômés de SF qui, lorsqu'ils n'ont pas effectué toute leur formation à l'université, sont aussi bien passés par une CPGE que par l'IUT.
Au total, ces statistiques descriptives montrent qu'il existe une grande diversité des parcours de formation chez les sortants de l'université titulaires d'un diplôme scientifique, particulièrement en sciences appliquées. Cette diversité des parcours de formation conduit-elle à une diversité des insertions professionnelles? 


\section{LE PARCOURS DE FORMATION : UNE VARIABLE DISCRIMINANTE DE L'INSERTION}

L'évaluation de l'insertion professionnelle est ici réalisée à l'aide de plusieurs indicateurs statistiques tirés de l'enquête Génération 98. La base de données choisie permet de travailler à partir d'informations quantitatives relativement riches sur le parcours scolaire puis professionnel d'un échantillon représentatif au niveau national des diplômés des filières scientifiques. Cette évaluation statistique gagnerait néanmoins à être complétée par des analyses qualitatives pour mieux comprendre les déterminants des modes de recrutement de jeunes scientifiques ou leur stratégie d'insertion professionnelle 5 .

L'impact du parcours est testé à l'aide de trois types d'analyse: sur quelques indicateurs d'insertion professionnelle, sur le salaire obtenu au bout de 3 ans et enfin en tenant compte de l'influence du parcours sur la spécialité du diplôme terminal.

\section{L'effet du parcours sur quelques indicateurs $d$ 'insertion professionnelle}

On a calculé, pour les diplômés de $2^{\mathrm{e}}$ (licencemaîtrise) et $3^{\text {e }}$ cycles (DEA et DESS), la valeur de quelques indicateurs classiques d'insertion professionnelle ${ }^{6}$, en fonction de leur parcours de formation (cf. tableau 2).

Le premier constat est que ces indicateurs varient significativement selon le type de parcours : le temps moyen d'accès au $1^{\text {er }}$ emploi varie du simple au double et le taux de chômage à 3 ans accuse des écarts de 9 points tandis que le taux d'emplois précaires $^{7}$ triple selon le parcours, et ce pour les deux cycles. La part d'emplois scientifiques, c'est-à-dire en relation avec la formation de ces jeunes ${ }^{8}$, passe de

\footnotetext{
${ }^{5}$ Fontanini (2001) montre par exemple que les diplômés scientifiques de haut niveau, notamment les filles, ont des stratégies professionnelles qui ne peuvent se résumer à des objectifs stricts de carrière.

${ }^{6}$ Voir Céreq 2001, "Quand l'école est finie... », pour la définition de ces indicateurs.

${ }^{7}$ Les emplois dont le contrat de travail est à durée déterminée (intérim, CDD, mesures jeunes...).

${ }^{8}$ Voir Béduwé et alii (2006) pour une discussion et une définition de « l'emploi scientifique ».
}

$42 \%$ à $81 \%$ selon les parcours et le diplôme, celui de cadres de $17 \%$ à $86 \%$. Enfin, la part de jeunes qui s'insèrent vite et durablement accuse des écarts de plus de $20 \%$, ce qui est très important. Le niveau de salaire moyen obtenu au bout de trois ans dépend lui aussi, à niveau de diplôme donné, du parcours de formation. Le parcours est donc, en première analyse et sur tous ces critères, une variable discriminante de l'insertion professionnelle.

Le deuxième constat est qu'on ne peut classer les parcours selon leur performance d'insertion: le parcours « $1^{\text {re }}$ année sélective + diplôme », qui correspond souvent à des poursuites d'étude à l'issue d'école d'ingénieurs ( $c f$. encadré 1) apparaît très bien placé sur la plupart des indicateurs et pour les deux niveaux de diplôme, mais les parcours via le BTS affichent également de très bons scores en $2^{\mathrm{e}}$ cycle, de même que ceux via le DUT en $3^{\mathrm{e}}$ cycle. À l'inverse, le parcours « filière sélective hors CPGE sans double diplôme » est souvent en bas de l'échelle pour la plupart des indicateurs, de même que les parcours purement universitaires. Il semble donc difficile d'établir une classification des parcours valable pour l'ensemble des indicateurs retenus.

On remarque cependant que les parcours présentant les meilleurs scores d'insertion sont ceux où les diplômés en sciences appliquées sont nombreux (tableau 1, dernière colonne). C'est le cas par exemple des parcours «IUT + DUT ». À l'inverse, les parcours purement universitaires, apparemment moins performants, sont majoritairement le fait de diplômés en sciences fondamentales. Ce constat est à rapprocher de résultats récurrents quant à l'insertion professionnelle des diplômés scientifiques qui montrent que, toutes choses égales par ailleurs, l'insertion des diplômés en sciences appliquées est plus rapide et plus satisfaisante que celle des diplômés en sciences fondamentales (Pottier, 1985 ; Béduwé et alii, 2007). L'explication souvent avancée est celle du cloisonnement de leurs marchés du travail respectifs. Les diplômés de sciences fondamentales qui n'ont pas obtenu le niveau de diplôme suffisant et/ou la qualification pour être enseignant ou chercheur - leur destination privilégiée - candidatent sur les emplois à caractère technologique du secteur privé (emplois d'ingénieur, de cadre technique, de technicien, désignés par emplois scientifiques dans 
Tableau 2

Type de parcours et indicateurs $d$ 'insertion

\begin{tabular}{|c|c|c|c|c|c|c|c|c|}
\hline & \multicolumn{7}{|c|}{ Indicateurs d'insertion : BAC +3 et 4 (Licence et Maîtrise) } & \multirow[b]{2}{*}{$\begin{array}{c}\text { Part de } \\
\text { diplômés } \\
\text { en SF } \\
(\%)\end{array}$} \\
\hline & $\begin{array}{c}\text { Temps } \\
\text { moyen } \\
d^{\prime} \text { 'accès au } \\
\text { ler emploi, } \\
\text { en mois }\end{array}$ & $\begin{array}{c}\text { Part de } \\
\text { jeunes } \\
\text { au chômage } \\
\text { au bout de } \\
3 \text { ans (\%) }\end{array}$ & $\begin{array}{c}\text { Part } \\
d^{\prime} \text { emplois } \\
\text { précaires } \\
\left({ }^{*}\right)(\%)\end{array}$ & $\begin{array}{c}\text { Part } \\
\text { d'emplois } \\
\text { de cadre } \\
(\%)\end{array}$ & $\begin{array}{l}\text { Trajectoire } \\
\text { type : accès } \\
\text { immédiat } \\
\text { et durable } \\
\text { à l'emploi (\%) }\end{array}$ & $\begin{array}{c}\text { Part } \\
\text { d'emplois } \\
\text { scientifiques } \\
\text { (\%) }\end{array}$ & $\begin{array}{l}\text { Salaire } \\
\text { moyen }\end{array}$ & \\
\hline $\begin{array}{l}\text { Universitaires } \\
\text { purs }\end{array}$ & 5,4 & 7,5 & 50 & 23 & 60 & 42 & $1344 €$ & 79 \\
\hline $\begin{array}{l}\text { IUT et DUT, } \\
\text { puis université }\end{array}$ & 3,1 & 4,3 & 35 & 36 & 74 & 63 & $1585 €$ & 30 \\
\hline $\begin{array}{l}\text { STS et BTS, } \\
\text { puis université }\end{array}$ & 2,7 & 1,4 & 27 & 25 & 87 & 74 & $1520 €$ & 39 \\
\hline $\begin{array}{l}\text { 1re année } \\
\text { sélective (hors } \\
\text { IUT et STS) ET } \\
\text { autre diplôme, } \\
\text { puis université }\end{array}$ & 2,8 & 0 & 14 & 46 & 87 & 63 & $1753 €$ & 30 \\
\hline CPGE puis fac & 3,7 & 2,1 & 22 & 49 & 65 & 56 & $1686 €$ & 62 \\
\hline $\begin{array}{l}\text { 1re sélective } \\
\text { (PCEM, IUT, } \\
\text { école ingénieur, } \\
\text { santé...) puis } \\
\text { université }\end{array}$ & 5,7 & 8,7 & 39 & 17 & 60 & 56 & $1422 €$ & 79 \\
\hline $\begin{array}{l}\text { Total des } \\
\text { diplômés } \\
\text { bac }+3,4 \\
\text { en Sciences }\end{array}$ & 4,5 & 5,7 & 41 & 27 & 67 & 52 & $1455 €$ & 62 \\
\hline
\end{tabular}

\begin{tabular}{|c|c|c|c|c|c|c|c|c|}
\hline & \multicolumn{7}{|c|}{ Indicateurs d'insertion : $\mathrm{BAC}+5$ (DEA et DESS) } & \multirow[b]{2}{*}{$\begin{array}{c}\text { Part de } \\
\text { diplômés } \\
\text { en SF } \\
(\%)\end{array}$} \\
\hline & $\begin{array}{c}\text { Temps } \\
\text { moyen } \\
\text { d'accès au } \\
\text { ler emploi }^{\text {er }}\end{array}$ & $\begin{array}{c}\text { Part de } \\
\text { jeunes } \\
\text { au chômage } \\
\text { au bout de } \\
3 \text { ans (\%) }\end{array}$ & $\begin{array}{c}\text { Part } \\
d^{\prime} \text { emplois } \\
\text { précaires } \\
(\%)\end{array}$ & $\begin{array}{c}\text { Part } \\
\text { d'emplois } \\
\text { de cadre } \\
\text { (\%) }\end{array}$ & $\begin{array}{l}\text { Trajectoire } \\
\text { type : accès } \\
\text { immédiat } \\
\text { et durable } \\
\text { à l'emploi (\%) }\end{array}$ & $\begin{array}{c}\text { Part } \\
\text { d'emplois } \\
\text { scientifiques } \\
\text { (\%) }\end{array}$ & $\begin{array}{l}\text { Salaire } \\
\text { moyen }\end{array}$ & \\
\hline $\begin{array}{l}\text { Universitaires } \\
\text { purs }\end{array}$ & $4,1(*)$ & 5,3 & 24 & 63 & 65 & 63 & $1826 €$ & 54 \\
\hline $\begin{array}{l}\text { IUT et DUT, } \\
\text { puis université }\end{array}$ & 2,3 & 4,8 & 13 & 79 & 80 & 81 & $1974 €$ & 30 \\
\hline $\begin{array}{l}\text { STS et BTS, } \\
\text { puis université }\end{array}$ & 2,7 & 2,5 & 29 & 65 & 73 & 75 & $1803 €$ & 20 \\
\hline $\begin{array}{l}\text { 1 re année } \\
\text { sélective (hors } \\
\text { IUT et STS) ET } \\
\text { autre diplôme, } \\
\text { puis université }\end{array}$ & 2,6 & 2,8 & 12 & 76 & 83 & 80 & $2144 €$ & 37 \\
\hline $\begin{array}{l}\text { CPGE puis } \\
\text { université }\end{array}$ & 4,3 & 4,9 & 19 & 86 & 67 & 63 & $1945 €$ & 57 \\
\hline
\end{tabular}


Tableau 2 (suite)

\begin{tabular}{|c|c|c|c|c|c|c|c|c|}
\hline & \multicolumn{7}{|c|}{ Indicateurs d'insertion : BAC +5 (DEA et DESS) } & \multirow[b]{2}{*}{$\begin{array}{c}\text { Part de } \\
\text { diplômés } \\
\text { en SF } \\
(\%)\end{array}$} \\
\hline & $\begin{array}{c}\text { Temps } \\
\text { moyen } \\
\text { d'accès au } \\
\text { 'er emploi }\end{array}$ & $\begin{array}{l}\text { Part de } \\
\text { jeunes } \\
\text { au chômage } \\
\text { au bout de } \\
3 \text { ans (\%) }\end{array}$ & $\begin{array}{c}\text { Part } \\
\text { d'emplois } \\
\text { précaires } \\
(\%)\end{array}$ & $\begin{array}{c}\text { Part } \\
\text { d'emplois } \\
\text { de cadre } \\
(\%)\end{array}$ & $\begin{array}{l}\text { Trajectoire } \\
\text { type : accès } \\
\text { immédiat } \\
\text { et durable } \\
\text { à l'emploi (\%) }\end{array}$ & $\begin{array}{c}\text { Part } \\
\text { d'emplois } \\
\text { scientifiques } \\
(\%)\end{array}$ & $\begin{array}{l}\text { Salaire } \\
\text { moyen }\end{array}$ & \\
\hline $\begin{array}{l}\text { 1 re sélective } \\
\text { (PCEM, IUT, } \\
\text { école ingénieur, } \\
\text { santé...) puis } \\
\text { université }\end{array}$ & 2,3 & 11,9 & 47 & 61 & 67 & 65 & $1931 €$ & 66 \\
\hline $\begin{array}{l}\text { Total des } \\
\text { diplômés } \\
\text { bac }+3,4 \\
\text { en Sciences }\end{array}$ & 3,4 & 5,1 & 22 & 69 & 70 & 68 & $1900 €$ & 47 \\
\hline
\end{tabular}

Source : Enquête Génération 98 à 3 ans, Céreq.

Sigles : DEA : diplôme d'études approfondies ; DESS : diplôme d'études supérieures spécialisées. DUT : diplôme universitaire de technologie (obtenu à l'issue d'une formation en IUT - Institut universitaire de technologie); BTS ; brevet de technicien supérieur (obtenu à l'issue d'une formation en STS - section de technicien supérieur); PCEM : première année commune aux formations de Médecin, Chirurgien Dentiste et Sage Femme; CPGE : classe préparatoire aux Grandes Écoles.

Note de lecture (*) : Les sortants diplômés d'un DEA ou d'un DESS après un parcours purement universitaire ont mis, en moyenne, 4,1 mois pour accéder à leur premier emploi, et au bout de 3 ans ils étaient 5,3 \% à connaître le chômage, 24 \% occupaient un emploi sous contrat précaire, $63 \%$ un emploi de niveau cadre, etc., et gagnaient en moyenne 1826 euros mensuels, primes incluses. On comptait $54 \%$ de spécialités de sciences fondamentales parmi eux.

(*) : Un emploi est qualifié de précaire si le contrat de travail n’est pas un contrat à durée indéterminée.

le tableau 2). Ils y entrent en compétition avec les diplômés en SA pour qui ces emplois constituent la cible professionnelle «naturelle» de leur formation. Cette concurrence, à niveau de diplôme donné, leur est défavorable.

Ces premiers résultats conduisent donc à se demander ce qui, de la spécialité ou du parcours, est à l'origine de cette diversité dans les performances d'insertion. Les diplômés en sciences appliquées doivent-ils leur meilleure insertion professionnelle à la spécificité des compétences conférées par leur diplôme final et/ou à celle de leurs parcours de formation ? Ceci incite à poursuivre l'analyse en utilisant des modèles « toutes choses égales par ailleurs ».

\section{Le parcours de formation a un impact sur les salaires}

Dans un premier temps, on a testé simultanément les effets du diplôme atteint, de sa spécialité et du type de parcours sur le salaire obtenu au bout de trois ans (tableau 3). Le niveau de salaire obtenu à ce moment est en effet un critère performant pour évaluer les écarts d'insertion de ces diplômés : pratiquement tous en emploi, ils se différencient surtout par leurs conditions de travail et leur métier, et donc leur niveau de rémunération.

À temps de travail donné, ancienneté dans l'emploi et durée d'expérience totale comparables, le diplôme obtenu est la variable qui discrimine le plus les salaires individuels. Certaines spécialités scientifiques ont également plus d'impact que d'autres, notamment l'informatique pour les SA et les mathématiques chez les SF. Lorsqu'on regroupe les spécialités en deux groupes, SF et SA, les diplômés en SF accusent une différence de salaire moyenne, toutes choses égales par ailleurs, de $12 \%$ du fait des avantages conférés par l'ensemble des spécialités appliquées. Ceci confirme les analyses antérieures et les résultats acquis à la section précédente.

Le parcours de formation a un effet sur le niveau de salaire, faible cependant en comparaison de celui du diplôme terminal et de sa spécialité. Toutefois, l'entrée dans le supérieur par une école d'ingénieur 
Tableau 3

Déterminants du salaire mensuel obtenu en avril 2001 par les diplômés universitaires en sciences Variable dépendante : logarithme du salaire net mensuel (euros) en avril 2001

\begin{tabular}{|c|c|c|}
\hline \multirow[b]{2}{*}{ Constante } & \multicolumn{2}{|c|}{$\begin{array}{l}\text { Ensemble des diplômés } \\
\text { universitaires scientifiques }\end{array}$} \\
\hline & 6,6 & 6,5 \\
\hline Homme & $+16 \%$ & $+14 \%$ \\
\hline Père Cadre ou Indépendant & $+4 \%$ & $+4 \%$ \\
\hline $\begin{array}{l}\text { Mère inactive } \\
\text { Mère Cadre ou indépendant } \\
\text { Mère autre CSP }\end{array}$ & $\begin{array}{l}\text { Ns } \\
\text { Ns } \\
\text { Ref. }\end{array}$ & $\begin{array}{l}\mathrm{Ns} \\
\mathrm{Ns} \\
\text { Ref. }\end{array}$ \\
\hline $\begin{array}{l}\text { Niveau du diplôme terminal } \\
\text { Echec au doctorat } \\
\text { DEA } \\
\text { DESS } \\
\text { Maîtrise } \\
\text { Licence }\end{array}$ & $\begin{array}{c}+29 \% \\
+24 \% \\
+25 \% \\
+9 \% \\
\text { Ref. }\end{array}$ & $\begin{array}{l}+29 \% \\
+25 \% \\
+25 \% \\
+11 \% \\
\quad \text { Ref. }\end{array}$ \\
\hline $\begin{array}{l}\text { Spécialité regroupée du diplôme terminal } \\
\text { Sciences fondamentales } \\
\text { Sciences appliquées }\end{array}$ & $\begin{array}{c}-0,12 \\
\text { Ref. }\end{array}$ & - \\
\hline $\begin{array}{l}\text { Spécialité détaillée du diplôme final } \\
\text { Maths } \\
\text { Physique } \\
\text { Chimie } \\
\text { Sciences nature et vie } \\
\text { Sciences de l'univers } \\
\text { Technologie } \\
\text { Électricité Électronique } \\
\text { Mécanique } \\
\text { Informatique }\end{array}$ & - & $\begin{array}{l}+16 \% \\
+11 \% \\
+10 \% \\
\text { Ref. } \\
\text { Ns } \\
+15 \% \\
+19 \% \\
+14 \% \\
+23 \%\end{array}$ \\
\hline $\begin{array}{l}\text { Parcours de formation supérieure } \\
\text { Universitaire } \\
\text { IUT et DUT puis fac } \\
\text { STS et BTS puis fac } \\
\text { 1re sélective (CPGE, école ingénieur, PCEM) ET autre diplôme, puis fac } \\
\text { CPGE puis fac } \\
\text { 1re sélective (PCEM, IUT, école ingénieur, santé...) puis fac }\end{array}$ & $\begin{array}{l}\text { Ref. } \\
\text { ns } \\
\text { ns } \\
+9 \% \\
+9 \% \\
\text { ns }\end{array}$ & $\begin{array}{l}\text { Ref. } \\
\text { ns } \\
\text { ns } \\
+9 \% \\
+9 \% \\
+5 \% *\end{array}$ \\
\hline Durée d'expérience acquise sur le marché du travail (en mois) & $+1 \%$ & $+1 \%$ \\
\hline Ancienneté dans l'emploi (en mois) & $+1 \%$ & $+0,8 \%$ \\
\hline $\begin{array}{l}\text { Temps de travail } \\
\text { Partiel } \\
\text { Temps plein }\end{array}$ & $\begin{array}{l}-23 \% \\
\text { Réf. }\end{array}$ & $\begin{array}{l}-21 \% \\
\text { Réf. }\end{array}$ \\
\hline $\mathrm{R}^{2}$ ajusté & 0,365 & 0,38 \\
\hline Effectif actifs occupés en avril 2001 & 1572 & 1572 \\
\hline
\end{tabular}

Source : Enquête Génération 98 à 3 ans, Céreq.

Note : Les coefficients sont significatifs au seuil de $1 \%$, sauf la CSP (catégorie socioprofessionnelle) du père qui l'est à $5 \%$.

Sigles : DEA : diplôme d'études approfondies ; DESS : diplôme d'études supérieures spécialisées. DUT : diplôme universitaire de technologie (obtenu à l'issue d'une formation en IUT - Institut universitaire de technologie); BTS ; brevet de technicien supérieur (obtenu à l'issue d'une formation en STS - section de technicien supérieur); PCEM : première année commune aux formations de Médecin, Chirurgien Dentiste et Sage Femme ; CPGE : classe préparatoire aux Grandes Écoles.

Note de lecture avec explication de la méthode : Il s'agit d'une régression linéaire du logarithme du salaire mensuel sur un ensemble de variable : Genre, CSP du père, Statut de la mère, etc. Elle permet de quantifier l'apport salarial des différentes modalités de chacune de ces variables. Ainsi les hommes gagnent en moyenne $16 \%$ de plus que les femmes dans le premier modèle (spécialité regroupée) et $14 \%$ de plus si l'on tient compte de la spécialité détaillée. 
ou par une CPGE apporte un bonus de $9 \%$. De même, lorsque l'on détaille la spécialité, le parcours «entrée dans l'enseignement supérieur par une filière sélective (hors CPGE) mais sans décrocher de diplôme » apporte un léger bonus de $5 \%$.

Les hommes gagnent, en moyenne et toutes choses égales par ailleurs, plus que les femmes, ce qui n'est pas nouveau. Au-delà d'un pur effet de discrimination salariale souvent dénoncé (Meurs, Ponthieux, 2000), ces écarts de salaire peuvent s'expliquer par des différences dans les emplois occupés ou les responsabilités détenues, pouvant être sujets à l'octroi de primes $^{9}$. Les jeunes d'origine sociale aisée (père cadre ou indépendant) enregistrent également une différence de salaire de $4 \%$. Ce résultat est également classique, quoique faible, compte tenu de l'importance des réseaux sociaux sur l'insertion professionnelle, mais on sait que le niveau de diplôme atteint capte une grande partie de cet effet et il est probable que c'est aussi le cas du parcours, voire de la spécialité du diplôme terminal.

\section{L'importance du parcours dans le « choix » de spécialité du diplôme terminal}

Les diplômés en SF obtiennent donc, en moyenne, de plus faibles rémunérations, à niveau de diplôme et parcours de formation donnés. L'impact du parcours sur le salaire, à diplôme et spécialité donnés, semble plus faible. Que deviennent ces résultats si le parcours et la spécialité finale ne sont plus considérés comme des critères indépendants mais bien comme dépendants l'un de l'autre, ce que suggèrent les données du tableau 1 ? C'est-à-dire si l'on tient compte, pour estimer les écarts de salaire, du fait que les diplômés de SF sont moins souvent issus d'IUT ou de STS que les diplômés en SA ? Dans ce cas-là, le désavantage observé des sciences fondamentales pourrait venir en partie de leur parcours de formation, ce qui peut se traduire, d'un point de vue économétrique, par l'existence d'un biais d'endogénéité10.

\footnotetext{
${ }^{9}$ Bien que plus faible, l'écart de salaire entre hommes et femmes persiste au sein des seuls emplois publics, toutes choses égales par ailleurs. ${ }^{10}$ La spécialité de formation est dite endogène si tout en influençant le salaire, elle est elle-même expliquée par d'autres variables du modèle comme le parcours de formation par exemple. Le biais vient de ce que l'on attribue à la spécialité un rôle dans l'analyse du salaire qui vient en partie du type de parcours.
}

La correction de ce biais est prise en compte par une méthode dite à «effet de traitement», proposée par Barnow, Cain et Goldberger (1981). Un premier modèle (probit) estime la probabilité qu'a un jeune scientifique de terminer ses études par un diplôme en sciences fondamentales plutôt qu'appliquées (tableau 4a). On a choisi de calculer cette probabilité en fonction des caractéristiques du parcours de formation, secondaire et supérieur, et de l'origine sociale des étudiants. On effectue ensuite une nouvelle régression du salaire (comme dans le tableau 3) qui tient compte de cette correction du bais supposé dans le « choix» de la spécialité finale (tableau 4 b). Le test présenté à la dernière ligne du tableau $4 \mathrm{~b}$ conduit à accepter l'hypothèse d'endogénéité sur la spécialité de formation : on a donc raison de séparer « choix de la spécialité » et « rendement de la spécialité ».

Les résultats du modèle de « choix » (probit) confirment, toutes choses égales par ailleurs, que la probabilité de sortir avec un diplôme en sciences fondamentales dépend largement du parcours de formation, secondaire et supérieur (tableau 4a). Avoir obtenu un DUT, un BTS ou un diplôme d'ingénieur diminue la probabilité de terminer ses études universitaires avec une spécialité de SF et augmente à l'inverse celle d'obtenir un diplôme terminal en SA. De même, l'obtention d'un baccalauréat scientifique (plutôt que technologique) et le passage par une seconde générale (plutôt que technologique) influent significativement sur la spécialité finale, en l'inscrivant dans un « choix » d'études générales opéré dès le secondaire. En revanche, le passage par une filière sélective, CPGE ou autre mais sans obtention de diplôme, n'a pas d'effet sur la spécialité finale du diplôme (alors que ces parcours expliquaient des écarts de salaire). Les variables concernant la profession des parents n'ont pas d'influence sur le choix de la spécialité, mais avoir une mère inactive diminue très légèrement la probabilité de choisir une spécialité de SF $(-9 \%)$. Enfin, les hommes ont, toutes choses égales par ailleurs, beaucoup moins de chances de terminer leurs études par une spécialité académique.

La fonction de gain associée montre un résultat essentiel: la prise en compte de l'endogénéité du choix de spécialité conduit à rendre non significatif l'effet de spécialité sur le salaire (tableau 4b). Autre- 
Tableau 4a

Estimation de la probabilité de sortir avec un diplôme universitaire en sciences fondamentales (plutôt qu'en sciences appliquées)

\begin{tabular}{|c|c|}
\hline & $\begin{array}{l}\text { Probabilité de sortir avec un diplôme } \\
\text { universitaire en sciences fondamentales }\end{array}$ \\
\hline Constante & $53 \% * \star \star$ \\
\hline Homme & $-24 \% * \star \star$ \\
\hline Père cadre ou indépendant & ns \\
\hline $\begin{array}{l}\text { Mère inactive } \\
\text { Mère cadre ou indépendant } \\
\text { Mère autre CSP }\end{array}$ & $\begin{array}{l}-9 \% * \\
\text { Ns } \\
\text { Ref. }\end{array}$ \\
\hline \multicolumn{2}{|l|}{ Parcours scolaire } \\
\hline Bac scientifique & $+9 \% * \star$ \\
\hline Retard au bac & Ns \\
\hline Seconde générale & $-26 \% * \star \star$ \\
\hline Avance en $6 \mathrm{e}$ & $\mathrm{Ns}$ \\
\hline Retard en 6e & ns \\
\hline $\begin{array}{l}\text { Parcours de formation supérieure } \\
\text { Universitaire } \\
\text { IUT et DUT puis fac } \\
\text { STS et BTS puis fac } \\
\text { 1re sélective (CPGE, école ingénieur, PCEM) ET autre diplôme, puis fac } \\
\text { CPGE puis fac } \\
\text { 1re sélective (PCEM, IUT, école ingénieur, santé...) puis fac }\end{array}$ & $\begin{array}{c}\text { Ref. } \\
-30 \% * * * \\
-16 \%{ }^{*} \\
-24 \%^{* * *} \\
\text { Ns } \\
\text { ns }\end{array}$ \\
\hline
\end{tabular}

Tableau 4b

Fonction de gains associée (correction de l'endogénéité)

\begin{tabular}{|l|c|}
\hline \multicolumn{1}{|c|}{$\begin{array}{c}\text { Variable dépendante : logarithme du salaire net mensuel (euros) en } \\
\text { avril 2001 }\end{array}$} \\
\hline Constante & 6,5 \\
\hline Homme & $23 \%$ \\
\hline Père cadre ou indépendant & $\mathrm{Ns}$ \\
\hline Mère cadre ou indépendant & $\mathrm{Ns}$ \\
\hline Niveau de diplôme terminal & $+29 \%$ \\
Sortants non diplômés de doctorat & $+25 \%$ \\
DEA & $+24 \%$ \\
DESS & $+10 \%$ \\
Maîtrise & Ref. \\
Licence & $0,15(0,12) \mathrm{ns}$ \\
\hline Spécialité du diplôme terminal & Ref. \\
Sciences fondamentales & \\
Sciences appliquées & \\
\hline
\end{tabular}




\section{Tableau 4b (suite)}

\begin{tabular}{|c|c|}
\hline $\begin{array}{l}\text { Variable dépendante : logarithme du salaire net mensuel (euros) en } \\
\qquad \text { avril } 2001\end{array}$ & \\
\hline $\begin{array}{l}\text { Parcours de formation supérieure } \\
\text { Universitaire } \\
\text { IUT et DUT puis fac } \\
\text { STS et BTS puis fac } \\
\text { 1 re sélective (CPGE, école ingénieur, PCEM) ET autre diplôme, puis fac } \\
\text { CPGE puis fac } \\
\text { 1 re sélective (PCEM, IUT, école ingénieur, santé...) puis fac }\end{array}$ & $\begin{aligned} & \operatorname{Ref} \\
+ & 12 \% \\
& \mathrm{Ns} \\
+ & 13 \% \\
+ & 14 \% \\
& \mathrm{Ns}\end{aligned}$ \\
\hline Durée d'expérience acquise sur le marché du travail & $+1 \%$ \\
\hline Ancienneté dans l'emploi & $+1 \%$ \\
\hline $\begin{array}{l}\text { Temps de travail } \\
\text { Temps plein } \\
\text { Partiel }\end{array}$ & $\begin{array}{l}\text { Réf. } \\
-23 \%\end{array}$ \\
\hline Rho & $-0,56(0,19)$ \\
\hline LR test $(\mathrm{Rho}=0)$, Chi2 $(1)=4.2$ & Rejeté à $2 \%\left({ }^{*}\right)$ \\
\hline
\end{tabular}

Source : Enquête Génération 98 à 3 ans, Céreq.

Note : Les coefficients sont au moins significatifs au seuil de $10 \%$.

Sigles : DEA : diplôme d'études approfondies ; DESS : diplôme d'études supérieures spécialisées. DUT : diplôme universitaire de technologie (obtenu à l'issue d'une formation en IUT - Institut universitaire de technologie); BTS ; brevet de technicien supérieur (obtenu à l'issue d'une formation en STS - section de technicien supérieur) ; PCEM : première année commune aux formations de Médecin, Chirurgien Dentiste et Sage Femme ; CPGE : classe préparatoire aux Grandes Écoles.

Note de lecture : Le salaire des jeunes passés par un IUT (plutôt que par l'université) sera majoré de $12 \%$, toutes choses égales par ailleurs et notamment à spécialité de formation donnée, tenant compte du fait que ce parcours via l'IUT diminue très fortement (30\%) la probabilité de terminer ses études avec un diplôme en SF.

${ }^{*}$ ) : « Rejeté » : La probabilité de se tromper en rejetant l'hypothèse d'une indépendance des deux équations est inférieure à $2 \%$.

ment dit, le désavantage salarial des diplômés en sciences fondamentales vient surtout de ce qu'ils n'ont pas les mêmes parcours de formation que les diplômés en sciences appliquées. À parcours identique, les spécialités de SF apparaissent aussi performantes que celles de SA. Ne pas tenir compte du lien existant entre un type de parcours et la spécialité finale du diplôme obtenu conduit à sous-estimer les gains des diplômés en SF.

Un autre résultat important concerne l'effet du parcours sur le niveau de salaire obtenu: même lorsque l'on tient compte du fait que le parcours de formation influence le choix de spécialité, il reste déterminant pour expliquer les rémunérations obtenues. Être passé par une CPGE, avoir obtenu un diplôme d'ingénieur à l'issue d'une filière sélective, ou encore un DUT sont les trois parcours qui permettent d'obtenir des gains majorés de plus de $10 \%$, à diplôme donné. On note que ces effets sont plus importants que dans le modèle précédent sans biais d'endogénéité. Ainsi, tenir compte de ce biais conduit à une substitution partielle entre effet de spécialité et effet de parcours: l'effet du parcours sur le salaire s'avère maintenant plus important que celui de la spécialité.

Les coefficients des autres variables (diplôme, ancienneté...) sont identiques à ceux obtenus sans correction de l'endogénéité, ce qui montre que leurs effets propres sont indépendants du choix de spécialité finale. On note en revanche un accroissement apparent de l'écart de salaire entre hommes et femmes.

Les trois parcours valorisés par le marché du travail (dans nos résultats) sont, on le constate, les plus sélectifs (CPGE, école d'ingénieurs); on peut dire également qu'ils correspondent à l'acquisition de diplômes cotés pour les savoirs qu'ils certifient (DUT, diplôme d'ingénieur). Les jeunes qui ont 
Tableau 5

Indicateurs de performance scolaire et origine sociale pour chaque parcours des diplômés 98 de l'université, en sciences

\begin{tabular}{|c|c|c|c|c|c|c|}
\hline \multirow[b]{2}{*}{$\begin{array}{c}\text { Parcours dans } \\
\text { l'enseignement supérieur }\end{array}$} & & \multicolumn{4}{|c|}{ Indicateurs de « performance » scolaire } & \multirow[b]{2}{*}{$\begin{array}{l}\text { Père } \\
\text { cadre } \\
(\%)\end{array}$} \\
\hline & & $\begin{array}{l}\text { Partd'étudiants } \\
\text { en avance } \\
\text { à l'entrée en } 6^{e}\end{array}$ & $\begin{array}{l}\text { Part de } \\
\text { secondes } \\
\text { générales }\end{array}$ & $\begin{array}{l}\text { Part de } \\
\text { Bac S }\end{array}$ & $\begin{array}{l}\text { Part d'étudiants } \\
\text { "à l'heure » } \\
\text { au Bac }\end{array}$ & \\
\hline Universitaires purs & $54 \%$ & $9 \%$ & $96 \%$ & $93 \%$ & $74 \%$ & 35 \\
\hline IUT et DUT, puis université & $20 \%$ & $11 \%$ & $82 \%$ & $84 \%$ & $62 \%$ & 24 \\
\hline STS et BTS, puis université & $8 \%$ & $12 \%$ & $55 \%$ & $38 \%$ & $65 \%$ & 22 \\
\hline $\begin{array}{l}\text { 1re année sélective (hors IUT et } \\
\text { STS) ET autre diplôme, puis fac }\end{array}$ & $5 \%$ & $24 \%$ & $87 \%$ & $77 \%$ & $88 \%$ & 33 \\
\hline CPGE puis université & $7 \%$ & $16 \%$ & $96 \%$ & $92 \%$ & $85 \%$ & 45 \\
\hline $\begin{array}{l}\text { 1re sélective (PCEM, IUT, école } \\
\text { ingénieur, santé...) puis } \\
\text { université }\end{array}$ & $6 \%$ & $18 \%$ & $89 \%$ & $86 \%$ & $75 \%$ & 33 \\
\hline $\begin{array}{l}\text { Total diplômés universitaires en } \\
\text { sciences }\end{array}$ & $\begin{array}{l}100 \%- \\
15968\end{array}$ & $11 \%$ & $89 \%$ & $85 \%$ & $72 \%$ & 32 \\
\hline
\end{tabular}

Source : Enquête Génération 98 à 3 ans, Céreq. En gris : valeurs supérieures à la moyenne.

Sigles : DUT : diplôme universitaire de technologie (obtenu à l'issue d'une formation en IUT - Institut universitaire de technologie) ; BTS ; brevet de technicien supérieur (obtenu à l'issue d'une formation en STS - section de technicien supérieur) ; PCEM : première année commune aux formations de Médecin, Chirurgien Dentiste et Sage Femme ; CPGE : classe préparatoire aux Grandes Écoles.

Exemple de lecture : les parcours purement universitaires représentent 54 \% des parcours de formation des diplômés en sciences de niveau supérieur à la licence. $9 \%$ des jeunes ayant eu ce type de parcours étaient en avance en $6^{\mathrm{e}}$.

connu ces parcours inscrivent donc dans leurs $\mathrm{CV}$ des éléments concernant leur formation et/ou font valoir des compétences qui, manifestement, sont appréciées des employeurs.

Qui sont ces jeunes? On peut montrer, à l'aide des mêmes données, que ces trois parcours correspondent également à des parcours scolairement valorisés qui attirent les bons élèves et les élèves d'origine sociale favorisée (tableau 5). On voit qu'effectivement les parcours marqués par un passage en CPGE sont, plus souvent qu'en moyenne, le fait : d'élèves en avance en $6^{\mathrm{e}}$, d'élèves issus de seconde générale, titulaires d'un bac $\mathrm{S}$, et à l'heure au bac (soit un score de 5 indicateurs de "performance scolaire» sur 5 dont la valeur est au-dessus de la moyenne). C'est un peu moins évident pour les parcours « $1^{\text {re }}$ sélective Et diplôme (ingénieur)» (2 indicateurs sur 5 au-dessus de la moyenne) et peu flagrant pour les parcours via
l'IUT (0 valeur au-dessus de la moyenne). Ainsi, le parcours, tout en étant révélateur d'un certain niveau d'excellence scolaire et/ou d'origine sociale favorisée, ne peut non plus se résumer à ces critères. On note à l'inverse que les parcours purement universitaires présentent de bons scores sur nos indicateurs de performance scolaire et d'origine sociale favorisée (4/5), alors qu'ils n'apportent pas de bonus en termes d'insertion professionnelle.

Une étape ultérieure consisterait à étudier les parcours eux-mêmes de manière à savoir qui emprunte telle ou telle filière et avec quel type de projet. C'est-à-dire remonter d'un cran dans l'analyse des biais d'endogénéité susceptibles de modifier les résultats d'insertion. On tiendrait compte ainsi du fait qu'un enfant de cadre a plus de chances d'accéder à une CPGE et donc de faire un parcours à la fois scolairement et professionnellement 
plus valorisé. Cela permettrait également d'affiner le rôle du genre dans ces « choix » de parcours. Mais, et c'est en ça que le résultat acquis est déjà très intéressant, il y a de grandes chances pour qu'un enfant de cadre aille plus souvent au bout de la filière CPGE, c'est-à-dire intègre une école et obtienne un diplôme terminal non universitaire. Ce qui le ferait sortir de notre champ d'analyse.

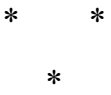

Le même diplôme universitaire en sciences peut être acquis à l'issue de parcours de formation différents dans l'enseignement supérieur. À l'aide des données de l'enquête Génération 98 du Céreq, on a montré que les titulaires du même diplôme universitaire scientifique ont effectivement commencé leurs études supérieures dans des filières différentes par leur mode de sélection (université, IUT, STS, CPGE, École d'ingénieur) et susceptibles de leur délivrer un diplôme à caractère technologique (DUT, BTS, ingénieur) relativement coté sur le marché du travail. Ces différences de parcours sont autant de "signaux» que l'individu peut inscrire dans son $\mathrm{CV}$, en complément de son diplôme terminal. Ils témoignent de compétences et d'expériences valorisables auprès des employeurs.

Cette diversité des parcours de formation entraîne une diversité des insertions professionnelles des diplômés en sciences, quel que soit le niveau finalement atteint (licence, maîtrise, DEA ou DESS). Avoir débuté ses études supérieures par une classe préparatoire aux Grandes Écoles, y compris sans avoir obtenu de diplôme d'ingénieur par la suite, ou par un DUT procure un avantage salarial (toutes choses égales par ailleurs) par rapport à ceux qui n'ont connu que l'université. Les parcours les plus valorisés scolairement ont donc un effet propre en termes de «performances » sur le marché du travail. Il semble que cet effet de parcours soit en partie capté par le type de spécialité, fondamental ou appliqué, du diplôme terminal. Ainsi, le niveau de salaire plus faible que l'on observe chez les diplômés en sciences fondamentales serait largement dû à des différences de parcours de formation. Tout se passe comme si la sélectivité des CPGE et des IUT, les compétences spécifiques attachées au DUT ou au diplôme d'ingénieur s'avéraient aussi probantes sur le marché du travail, sinon plus, que la spécificité des compétences certifiées par la spécialité du diplôme terminal. On sait du reste que ces parcours avec passages par des filières sélectives correspondent à des stratégies individuelles d'étudiants qui ont compris comment «utiliser » le système de formation supérieure quand d'autres au contraire le subissent (Blöss et Erlich, 2000 ; Beaud, 2002) ${ }^{11}$.

Ces résultats sont un premier test qui demande à être confirmé sur d'autres données et même étendu à d'autres domaines de l'enseignement supérieur. Tels quels, ils s'inscrivent dans le débat actuel sur la déconnexion croissante qui s'opère entre parcours de formation et diplôme et les conséquences que cela engendre pour l'évaluation de l'insertion professionnelle.

Dans cette perspective, le caractère homogénéisateur du diplôme, a fortiori celui du diplôme terminal, visà-vis de l'accès à l'emploi, n'est-il pas remis en cause? Les analyses conduites par le Céreq depuis quarante ans ont montré le rôle déterminant du diplôme, et même du diplôme de niveau le plus élevé, sur la qualité du processus d'insertion professionnelle et les rémunérations associées. Plus le niveau de formation est élevé dans des champs professionnels bien identifiés, plus l'insertion se déroule dans de bonnes conditions. Ces analyses reposent implicitement sur l'idée d'une certaine homogénéité des connaissances et des compétences des titulaires d'un même diplôme. L'idée qu'il existe de «meilleurs» parcours que d'autres remet en cause cette hypothèse, en introduisant une différence entre les titulaires d'un même diplôme. Un employeur peut préférer embaucher un diplômé de licence ou maîtrise acquis après un DUT plutôt qu'un

\footnotetext{
${ }^{11}$ Pour comprendre les difficultés d'orientation d'une partie des bacheliers issus de la démocratisation scolaire, Stéphane Beaud (2002, p. 138) analyse le cas de Gundur, un bachelier issu de lycée de ZEP (zone d'éducation prioritaire), qui fait un dossier d'inscription en BTS et qui échoue lors de l'entretien de sélection " faute de pouvoir s'identifier à l'avance à l'image sociale du BTS et aussi par manque de ressources sociales ». Stéphane Beaud conclut, p. 140, que "Le décalage est flagrant entre les attentes sociales, les dispositions souhaitées et souhaitables de l'institution scolaire - qui elle-même anticipe sur celles de l'entreprise et des offreurs d'emploi - et l'attitude indéterminée de Gundur qui apparait aux antipodes de celles attendues d'un futur technicien ou col blanc». À la rentrée, Gundur se retrouve à la fac, envisagée comme « roue de secours ».
} 
étudiant ayant le même diplôme obtenu après un DEUG, ou inversement. L'hypothèse d'indifférence de l'employeur aux éléments du parcours qui a précédé l'obtention du diplôme, hypothèse implicite des analyses de performance d'insertion basées sur le diplôme terminal, paraît alors difficilement tenable. Le parcours de formation entre dans la nature et le signalement des compétences acquises, et son influence sur le processus d'insertion professionnelle semble beaucoup plus complexe, avec de possibles effets de substitution, selon les arbitrages du marché.

Cette évolution n'est pas sans conséquences sur l'utilisation qui peut être faite des indicateurs statistiques rendant compte des performances d'un diplôme sur le marché du travail pour aider les jeunes dans leur orientation. Le présent travail suggère que focaliser l'attention des universités sur les performances d'insertion du seul diplôme terminal, sans prendre en compte les éléments du parcours préalable, serait une simplification hâtive. Le niveau de diplôme terminal est un facteur majeur de l'insertion, mais à diplôme égal, le parcours universitaire composé de formations souvent dispensées en dehors de l'université joue un rôle important. Est-ce la responsabilité des universités si leurs diplômés qui obtiennent les meilleures performances d'insertion sur le marché sont ceux... qui n'ont pas commencé leur parcours supérieur par l'université ?

Faire en sorte que les indicateurs statistiques publiés pour chaque diplôme par chaque université aident les jeunes à choisir leur formation relève d'un double défi : identifier les indicateurs capables de rendre compte de la complexité des relations entre formations, diplômes et emplois telles qu'elles se tissent réellement sur le marché du travail d'une part, donner les clés d'interprétation des valeurs fournies par ces indicateurs et les conseils qui en découlent en matière d'orientation d'autre part. Le premier point est relativement technique. Ce travail s'inscrit dans cette perspective. Le second passe par l'intermédiation d'acteurs (décideurs et usagers des formations supérieures) capables d'analyser toutes ces informations, y compris de manière prospective, et de les mettre à disposition des jeunes pour les aider à bien choisir leur formation supérieure et leur parcours de formation.

\section{Bibliographie}

Adangnikou N. (2007), Une évaluation de l'efficacité de l'enseignement supérieur français : le cas des classes préparatoires scientifiques, Thèse de doctorat sous la direction de M. Duru-Bellat et J.-J. Paul, Université de Bourgogne, 339 p.

Barnow B., Cain G., Goldberger A. (1981), "Issues in the Analysis of Selectivity Bias", in E. Stromsdorfer and G. Farkas (eds.), Evaluation Studies Review Annual, 5, Beverly Hills, Calif.

Beaud S. (2002), 80\% au bac. Et après?... Les enfants de la démocratisation, La Découverte, Paris.

Béduwé C., Fourcade B., Giret J.-F., Moullet S. (2006), «Les filières scientifiques et l'emploi»,
Dossier Insertion, Éducation et Société du MENR, $\mathrm{n}^{\circ} 177,230 \mathrm{p}$, septembre.

Béduwé C., Fourcade B., Giret J.-F., Moullet S. (2007), «Études scientifiques et marché du travail : éléments de réflexion sur la crise des sciences », L'Orientation scolaire et professionnelle, vol. 36/ $\mathrm{n}^{\circ}$ 4, décembre, pp. 503-532.

Blöss T., Erlich V. (2000), « Les nouveaux acteurs de la sélection universitaire. Les bacheliers technologiques en question ", Revue française de sociologie, $\mathrm{n}^{\circ} 41,4$, pp. 747-775.

Bouffartigue P., Gadea C. (1997), «Les ingénieurs français. Spécificités nationales et dynamiques 
récentes d'un groupe professionnel », Revue française de sociologie, XXXVIII, pp. 301-326.

Céreq (2002), Quand l'école est finie, 75 p.

Charlot A., Pottier F. (1989), « Dix ans d'insertion des diplômés universitaires ", Formation Emploi $\mathrm{n}^{\circ} 25$, janvier-mars, pp. 3-18.

Dauty F., Lemistre P. (2008), «Diversité des parcours des niveaux $\mathrm{V}$, une composante des effets de spécialité ?, » in Cart B. et Alii (Eds), Derrière les diplomes et certifications, les parcours de formation et leurs effets sur les parcours d'emploi, ReliefCéreq, n 24 , pp. 287-298.

Duhautois R., Maublanc S. (2005), Les carrières des chercheurs en entreprise, Rapport de recherche du CEE $n^{\circ} 25$.

Dupray A., Recotillet I. (2005), «Quelle adéquation à l'emploi pour les jeunes issus d'itinéraires scolaires atypiques? ", in J.-F. Giret, A. Lopez et J. Rose, Quelles formations pour quels emplois ?, La Découverte, pp. 203-220.

Eymard-Duvernay F., Marchal E. (2000), «Qui calcule trop finit par déraisonner: les experts du marché du travail », Sociologie du Travail, vol. 42, pp. 411-432.

Fontanini C. (2001), «Élèves ingénieurs : aspirations et projets de vie. Une question de genre », Carrefours de l'éducation, 2001/1, n ${ }^{\circ} 11$, pp. 52-65.

Forsé M. (2001), «Rôle spécifique et croissance du capital social », Revue de l'OFCE, janvier, $\mathrm{n}^{\circ} 76$, pp. 189-216.

Garcia-Aracil A., Van der Velden R. (2008), "Competencies for young european higher education graduates: labour market mismatches and their payoffs", Higher Education, vol. 55, n 2, pp. 219239.

Giret J.-F., Moullet S. (2008), «Une analyse de la professionnalisation des formations de l'enseigne- ment supérieur à partir de l'insertion de leurs diplômés », Céreq, Net.doc n 35 .

Giret J.-F. (2003), « La diversité des parcours universitaires influence-t-elle l'insertion professionnelle des diplômés de l'enseignement supérieur?», in Parcours étudiants : de l'enseignement supérieur au marché du travail, Groupe de travail sur l'enseignement supérieur, Relief-Céreq n ${ }^{\circ}$ 1, pp. 75-86.

Hilmer M.J. (2006), "Does the return to university quality differ for transfer students and direct attendees?", Economics of Education Review, 19, 25(4), pp. 47-61.

Lanciano-Morandat C. (1996), «Les ingénieurs des Sociétés de service et d'ingénierie informatique. Espace productif et professionnalité », Formation Emploi, $\mathrm{n}^{\circ}$ 55, pp. 77-89.

Marchal E., Rieucau G. (2005), « Candidat de plus de 40 ans, non diplômé ou débutant s'abstenir », Connaissance de l'emploi, CEE, $\mathrm{n}^{\circ} 11$, janvier, $4 \mathrm{p}$.

Meng C., Heijke H. (2005), Student time allocation, the learning environment and the acquisition of competencies, ROA-RM-2005/1E, Research Centre for Education and the Labour Market, Maastricht.

Meurs D., Ponthieux S. (2000), «Une mesure de la discrimination dans l'écart de salaire entre homes et femmes ", Économie et Statistiques, 337-338, pp.135-158.

Pottier F. (1985), « L'avenir des diplômés de l'enseignement supérieur scientifique », Formation Emploi $\mathrm{n}^{\circ} 10$, avril-juin, pp. 3-22

Pottier F. (1996), « Les ingénieurs du CNAM : quels devenirs? », Formation Emploi, n 55, pp. 59-75.

Van der Velden R., Wolbers M. (2007), "How much does education matter and why? The Effects of Education on Socio-economic Outcomes among School-leavers in the Netherlands", European Sociological Review, February 2007, 23, pp. 65-80. 


\title{
Résumé
}

\section{De l'influence du parcours de formation sur l'insertion : le cas des diplômés scientifiques}

\author{
Catherine Béduwé, Bernard Fourcade et Jean-François Giret
}

Un même diplôme universitaire peut être acquis après différents parcours de formation dans l'enseignement supérieur. Cette diversité des parcours de formation conduitelle à une diversité des insertions professionnelles ? L'hypothèse a été testée auprès des diplômés de l'université en sciences à l'aide des données de l'enquête Génération 98 du Céreq. Dans un premier temps, on montre que le parcours joue un rôle relativement secondaire en comparaison du diplôme terminal et de sa spécialité. Le parcours devient déterminant, et supplante même la spécialité, dès lors que l'on tient compte des liens qu'il entretient avec la spécialité finale du diplôme. Ces premiers résultats remettent en cause le rôle homogénéisateur du diplôme final et, ce faisant, alimentent le débat en cours sur les conséquences d'une déconnexion croissante entre formation et certification.

\section{Mots clés}

Insertion professionnelle, diplôme, enseignement supérieur, science, enquête génération 98, cheminement universitaire, filière de formation, salaire

Journal of Economic Literature: J 23 